\title{
Stachybotrys mycotoxins: from culture extracts to dust samples
}

\author{
Ina Došen ${ }^{1} \cdot$ Birgitte Andersen $^{1}$ - Christopher B. W. Phippen ${ }^{1} \cdot$ Geo Clausen ${ }^{2}$. \\ Kristian Fog Nielsen ${ }^{1}$
}

Received: 25 February 2016 /Revised: 28 April 2016 / Accepted: 17 May 2016 /Published online: 2 June 2016

(C) The Author(s) 2016. This article is published with open access at Springerlink.com

\begin{abstract}
The filamentous fungus Stachybotrys chartarum is known for its toxic metabolites and has been associated with serious health problems, including mycotoxicosis, among occupants of contaminated buildings. Here, we present results from a case study, where an ultra-high performance liquid chromatography-tandem mass spectrometry (UHPLC-MS/ MS) method was developed for known and tentatively identified compounds characterized via UHPLC-quadruple timeof-flight (QTOF) screening of fungal culture extracts, wall scrapings and reference standards. The UHPLC-MS/MS method was able to identify 12 Stachybotrys metabolites, of which four could be quantified based on authentic standards and a further six estimated based on similarity to authentic standards. Samples collected from walls contaminated by $S$. chartarum in a water-damaged building showed that the two known chemotypes, $\mathrm{S}$ and $\mathrm{A}$, coexisted. More importantly, a link between mycotoxin concentrations found on contaminated surfaces and in settled dust was made. One dust sample, collected from a water-damaged room, contained $10 \mathrm{pg} / \mathrm{cm}^{2}$ macrocyclic trichothecenes (roridin E). For the first time, more than one spirocyclic drimane was detected in dust. Spirocyclic drimanes were detected in all 11 analysed dust
\end{abstract}

Electronic supplementary material The online version of this article (doi:10.1007/s00216-016-9649-y) contains supplementary material, which is available to authorized users.

Kristian Fog Nielsen

kfn@bio.dtu.dk

1 Section for Eukaryotic Biotechnology, Department of Systems Biology, Technical University of Denmark, Søltofts Plads, 2800 Lyngby, Denmark

2 International Centre for Indoor Environment and Energy, Department of Civil Engineering, Technical University of Denmark, Nils Koppels Allé, 2800 Lyngby, Denmark samples and in total amounted to $600 \mathrm{pg} / \mathrm{cm}^{2}$ in the waterdamaged room and $340 \mathrm{pg} / \mathrm{cm}^{2}$ in rooms adjacent to the water-damaged area. Their wide distribution in detectable amounts in dust suggested they could be good candidates for exposure biomarkers.

Keywords Stachybotrys · Mycotoxin · Dust · Spirocyclic drimane $\cdot \mathrm{QTOF} \cdot \mathrm{QqQ}$

\section{Introduction}

Fungal spores are ubiquitous in indoor environments, and the growth of mould in buildings can often lead to negative health effects such as skin rashes, headaches, dizziness and chronic fatigue of the occupants [1-3]. Although the presence of moulds in the indoor environment is generally undesirable, some species, such as Stachybotrys chartarum and Chaetomium globosum, are considered more problematic [3]. S. chartarum is known for its production of toxic metabolites that have been connected to the extensive occurrence of negative health effects $[1,4]$ and possibly also linked to idiopathic pulmonary haemosiderosis in babies $[5,6]$. Stachybotrys has historically been shown to be responsible for severe toxicoses in farm animals fed with contaminated hay $[7,8]$.

S. chartarum can be found in two chemotypes, S and A, both sharing the same morphology, but differing in some of the metabolites they produce [9]. Chemotype $\mathrm{S}$ produces macrocyclic trichothecenes (satratoxins, verrucarins and roridins; Fig. 1), which are some of the most cytotoxic compounds currently known [10]. Chemotype A produces atranones and their precursors, dolabellanes, together with the simple nonmacrocyclic trichothecene, trichodermin [9]. Both chemotypes also produce many metabolites belonging to the 


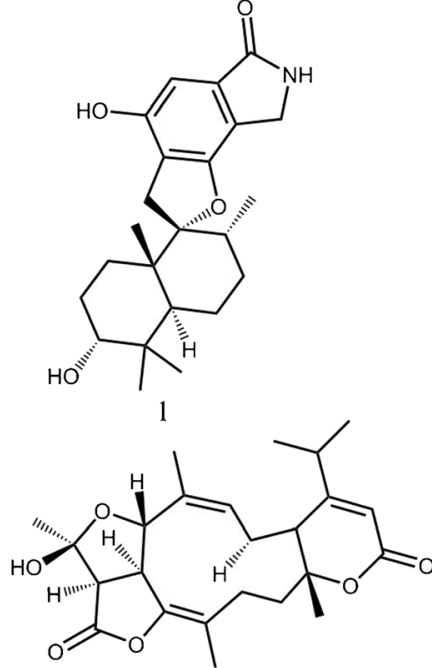

5

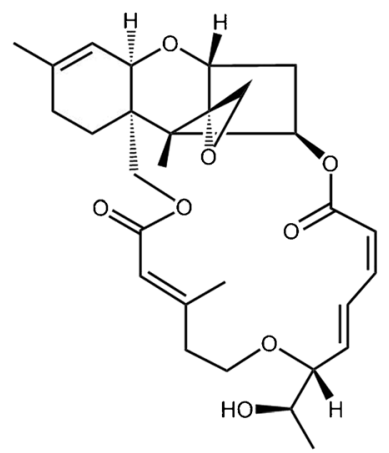

9

10

Fig. 1 Secondary metabolites and mycotoxins produced by S. chartarum: spirocyclic drimanes produced by both chemotypes (1 stachybotrylactam, 2 stachybotryamide, 3 stachybotrydial, 4 Mer-NF5003-B), atranones and their precursors characteristic of chemotype A

spirocyclic drimane family [11], in much greater quantity than the trichothecenes and antranones [12-14]. Chemotype A induces highly inflammatory effects both in vivo and in vitro $[10,14,15]$, but the causative agents for this have not been revealed yet. It is possible that the observed inflammatory effects are caused by the drimanes, atranones, or other compounds present in both types but that these effects may be concealed in the chemotype $\mathrm{S}$ by the highly cytotoxic macrocyclic trichothecenes [14]. Furthermore, Stachybotrys chlorohalonata is also found in buildings, but chemically, it can currently not be differentiated from $S$. chartarum chemotype A [9].

S. chartarum requires high water activity $(0.98)$ for growth [16], which in practice for the indoor environment means water ingress rather than high levels of condensation. $S$. chartarum is mainly observed on materials with high cellulose content, such as gypsum wallboard, straw, wallpapers and some insulation materials $[13,17]$. Spores of $S$. chartarum are produced in sticky slime heads [18] and do not easily<smiles>C[C@H]1CC[C@]2(C)C(C)(C)[C@@H](O)CC[C@]2(C)[C@]12Cc1c(O)cc(C=O)c(C=O)c1O2</smiles>

3

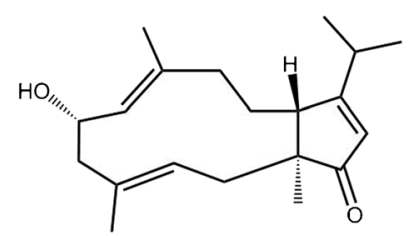

7

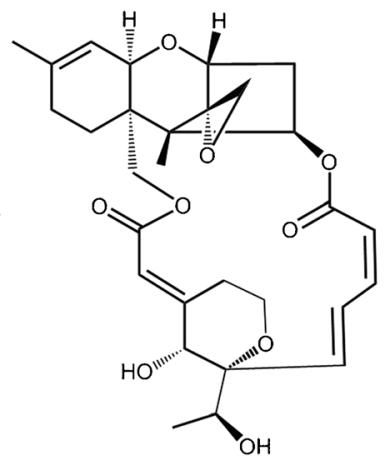

11<smiles>C[C@@H]1CC[C@]2(C)C(C)(C)[C@@H](O)[C@@H](O)C[C@]2(C)[C@]12Cc1c(O)cc(C=O)c(C=O)c1O2</smiles>

4

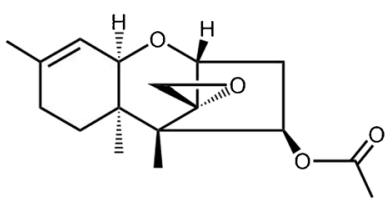

8

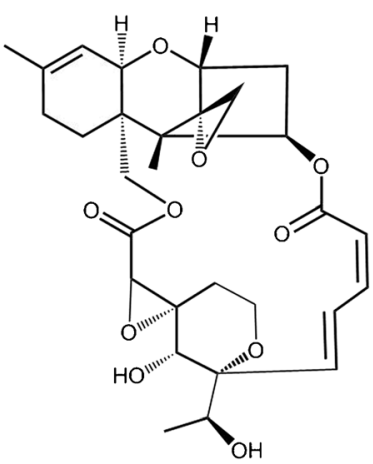

12
(5 atranone A, 6 atranone $\mathrm{B}, 7$ and 6 hydroxydolabella- $3 E, 7 E, 12$-trien14-one, 8 simple trichothecene trichodermin) and macrocyclic trichothecenes characteristic of chemotype $\mathrm{S}$ ( 9 roridin $\mathrm{E}, 10$ roridin $\mathrm{L} 2$, 11 satratoxin $\mathrm{H}, 12$ satratoxin $\mathrm{G})$

become airborne when they are moist, making detection in the air difficult. Hence, exposure mainly occurs from dry, disintegrating spores and mycelium as micro-particles [19-21].

Based on the database Antibase2012, as well as other sources [10, 12, 22-24], there are currently around 140 compounds described from Stachybotrys spp. Only one of these compounds, stachybotrylactam, is commercially available whilst satratoxins $\mathrm{H}$ and $\mathrm{G}$ have also been partially available. A few authors have developed trace analytical methods including these three compounds, based on liquid chromatography-tandem mass spectrometric (LC-MS/MS) detection [25-27]. The macrocyclic trichothecenes can also be analysed by immunochemical methods [28, 29] or via hydrolysis, derivatization and GC-MS $[11,30]$. Although a large amount of attention has been placed on the presence of Stachybotrys mycotoxins in the indoor environment, attempts to identify these toxics in the dust usually fail, as do attempts to correlate amounts found on contaminated surfaces with 
amounts found in dust. This failure is especially true in cases where the contaminant is $S$. chartarum chemotype A or $S$. chlorohalonata, as none of existing methods targets atranones or their precursors, the dolabellanes. Furthermore, all the major spirocyclic drimanes, except for stachybotrylactam, are not covered by the existing targeted LC-MS/MS methods.

Earlier analytical methods for Stachybotrys metabolites, mainly based on LC separation with UV/vis diode array detection (DAD) $[9,12,31]$, clearly showed that drimanes were the dominant compounds in the analysed extracts. In order to separate drimanes from atranones or macrocyclic trichothecenes, samples were purified by normal phase solid phase extraction on PEI silica [12]. The introduction of LC in combination with high-resolution mass spectrometry (HRMS) has allowed both time-of-flight (TOF) and Orbitrap instruments to be used routinely for fungal metabolite profiling [32, 33]. One of the advantages of LC-HRMS over UV/vis is that it is easier to distinguish co-eluting compounds using extracted ion chromatograms [34]. Despite this advantage, many fungal metabolites possess the same elemental composition, and even more specific/accurate identification than HRMS is required to distinguish similar compounds. This greater specificity of identification can be obtained from MS/HRMS either by using standardized MS/HRMS libraries with three fixed fragmentation energies (e.g. 10, 20 and $40 \mathrm{eV}$ as introduced by Agilent Technologies) $[35,36]$ or more energies, as on ThermoFisher instruments [37].

Our recent work [36] presented the use of MS/HRMS libraries in fungal drug discovery, and we are currently expanding our existing library with Stachybotrys metabolites. Agilent Technologies state that fragmentation energies are standardized across their instruments, including triple quadrupole instruments (QqQ). Consequently, we hypothesize that multiple reaction monitoring (MRM) could be predicted directly from the MS/HRMS library, not only including fragment ions but also the collision energies. In culture extracts analysed by UHPLC-DAD-HRMS, we observed that most of the spirocyclic drimanes ionized very well compared to their apparent intensity in the UV/vis chromatogram. Considering that QqQ instruments are typically 5-50-fold more sensitive than HRMS instruments, we hypothesized that by mapping all the major spirocyclic drimanes from Stachybotrys cultures and infected material samples and transferring the method to a QqQ instrument, we would stand a greater chance of detecting these in settled dust in Stachybotrys-infected buildings than other metabolites such as the trichothecenes.

In this case study, we tested these hypotheses by identifying promising $S$. chartarum biomarkers using UHPLC-QTOF in extracts from pure fungal cultures and cotton tip swabs from infected gypsum wallboards and further transferring detection of these directly to a UHPLC-QqQ method. This method was combined with standards of several macrocyclic trichothecenes, several atranones, one commercially available spirocyclic drimane and the simple trichothecene trichodermin.

\section{Materials and methods}

\section{Chemicals and standards}

All solvents, including water for LC-MS analyses, were LCMS grade; chemicals were analytical grade and were purchased from Sigma-Aldrich (Steinheim, Germany), if not otherwise stated. Standard of stachybotrylactam was purchased from Enzo (Exeter, UK). All other standards were available in-house, donated by other research groups or purified from different in-house projects [36]. Standards for quantification purposes were weighed and dissolved either in pure acetonitrile (trichodermin ( $\geq 95 \%$ pure HPLC)), acetonitrile/water $(90: 10, v / v)$ (roridin E ( $\geq 90 \%$ pure HPLC)) or pure methanol (stachybotrylactam ( $\geq 95 \%$ pure HPLC); satratoxin $\mathrm{H}(\geq 90 \%$ pure HPLC)). Individual stock solutions were kept in $-20{ }^{\circ} \mathrm{C}$. Prior to analysis, stock solutions were brought to room temperature, thoroughly mixed and used to prepare multi-analyte standard solutions in triplicates, at five (satratoxin $\mathrm{H}$, roridin E) or six (stachybotrylactame, trichodermin) different levels with concentrations of 10,100, 300, 1000, 3000 and 10, $000 \mathrm{ng} / \mathrm{mL}$.

\section{Sampling of fungal biomass and settled dust}

All samples in this case study were collected from a waterdamaged kindergarten in the Greater Copenhagen area. A broken concealed water pipe in a ground floor bathroom had resulted in fungal contamination in the internal structures of the wall ( $>80 \%$ of the surface covered with fungal growth). The whole ground floor was closed off due to the high number of children $(>50 \%)$ reporting sickness.

The wall was opened and fungal biomass samples were collected from the gypsum wallboards in the bathroom. Each sample was taken from an approximate surface area of $1 \mathrm{~cm}^{2}$ using a sterile cotton tip in a screw-cap plastic tube (Qtips) or by scraping off small pieces of infected surface materials. The collected samples were kept in tubes in a ventilated room at $10^{\circ} \mathrm{C}$ until analysis.

Settled dust samples were collected from all available surfaces (shelves, tables, fridge, tops of the hanging lamps) and other places (excluding the floor) that were regularly cleaned. Each sample was taken from an approximate surface area of $45 \times 45 \mathrm{~cm}$ using a clean precision Kimwipes ${ }^{\circledR}$ Lite wipe (Kimberly-Clark, GA, USA). Dust was collected both in the bathroom where the water damage occurred and in neighbouring rooms. The collected dust together with the wipes were placed in $50-\mathrm{mL}$ falcon tubes (VWR, 
Philadelphia, USA), and the tubes were closed and kept in a ventilated room at $10^{\circ} \mathrm{C}$ until analysis.

Tape preparations for phase-contrast microscopy $(\times 200$ and $\times 400$ ) were taken directly from the mould-infected area. This was done by gently pressing transparent adhesive tape to the infected surface and mounting it on a microscope slide in a drop of Shear's mounting fluid [18]. Scrapings were used for fungal identification by metabolite profiling [9] where obtained metabolite profiles from scraped contaminated material were compared to metabolite profiles of in-house indoor Stachybotrys strains. Four S. chartarum (two chemotype S and two chemotype A) and two $S$. chlorohalonata strains were used for metabolite profiling (Table 1). Agar media used for pure strain inoculation were malt extract agar (MEA) and potato dextrose agar (PDA) [18]. After inoculation, the strains were incubated in darkness at $25^{\circ} \mathrm{C}$ for $1-2$ weeks, after which sampling for metabolite profiling was performed (PDA and MEA extracts).

\section{Extraction of pure cultures, biomass and dust}

Extraction of pure agar cultures was performed using a microscale extraction method modified for Stachybotrys metabolites [38]. Three agar plugs (6 mm ID) were cut from a 15day-old colony from each agar medium (PDA or MEA) and placed in a 2-mL screw-top vial. $1.0 \mathrm{~mL}$ of extraction solvent (ethyl acetate/dichloromethane/methanol $(3: 2: 1, v / v / v)$ containing $1 \%$ formic acid) was added to each vial, and the plugs were extracted by sonication for $60 \mathrm{~min}$. The extracts were further treated as described below for other types of samples.

For fungal biomass collected with Q-tips from the bathroom, the cotton tip of a swab was carefully cut using a disposable scalpel and transferred to $15-\mathrm{mL}$ falcon tube. For the settled dust collected on wipes, the wipes were transferred to 50-mL falcon tubes.

To each Falcon tube was added either $15 \mathrm{~mL}$ (dust samples) or $2 \mathrm{~mL}$ (biomass samples) extraction solvent (acetoni trile:water $(75: 25 v / v)$ containing $1 \%$ formic acid). The tubes were placed in an ultrasonication bath for $60 \mathrm{~min}$ and then centrifuged at $4000 \mathrm{~g}$ for 2 min after which the liquid was transferred to a clean tube and evaporated to dryness under a gentle stream of $\mathrm{N}_{2}$. Thereafter, the samples were re-dissolved in $400 \mu \mathrm{L}$ solvent (acetonitrile:Milli-Q water $(75: 25 \nu / v)$ with $1 \%$ formic acid) and centrifuged (15 min, 15,000g). The supernatant was used directly for chemical analysis, which was conducted within 2 days, where the samples were stored at $-20{ }^{\circ} \mathrm{C}$ prior to transfer to the autosampler held at $5{ }^{\circ} \mathrm{C}$.

\section{UHPLC-DAD-QTOF analysis}

Metabolite profiling of extracts of pure cultures, biomass and dust samples collected in the water-damaged kindergarten was performed using ultra-high performance liquid chromatography-diode array detection-quadrupole time-offlight mass spectrometry (UHPLC-DAD-QTOFMS) as described by Dosen et al. [39] using an Agilent Infinity 1290 UHPLC system (Agilent Technologies, Santa Clara, CA, USA) coupled to an Agilent 6545 QTOF MS equipped with a Dual Jet Stream electrospray ion source. The system was equipped with a diode array detector scanning in the range 200-640 nm 20 times/s [36]. Separation was performed on an Agilent Poroshell 120 Phenyl-Hexyl column $(2.1 \times 150 \mathrm{~mm}, 2.7 \mu \mathrm{m})$ at $60{ }^{\circ} \mathrm{C}$ at a flow of $0.35 \mathrm{~mL} / \mathrm{min}$. A linear solvent gradient, consisting of A: $20 \mathrm{mmol} / \mathrm{L}$ formic acid in water and B: $20 \mathrm{mmol} / \mathrm{L}$ formic acid in acetonitrile was used, graduating from 10 to $100 \%$ B within 15 min, held for 2 min, followed by returning to $10 \%$ in $0.1 \mathrm{~min}$ and remaining for $3 \mathrm{~min}$, giving a total analysis time of $20 \mathrm{~min}$ [36]. Lock mass solution in 80/20 methanol/water ( $v / v)$ was infused in the second sprayer using an extra LC pump at a flow of $1.5 \mathrm{~mL} / \mathrm{min}$ which was subsequently split 1:100, delivering $15 \mu \mathrm{L}$ to the MS of $10 \mu \mathrm{M}$ hexakis(2,2,3,3tetrafluoropropoxy)phosphazene (Apollo Scientific Ltd, Cheshire, UK) as lock mass. Other MS parameters, including information on automated data-dependant MS/HRMS (autoMS/HRMS), can be found in [36]. Samples were largely analysed in $\mathrm{ESI}^{+}$mode.

Identification of secondary metabolites was performed using a combination of the following approaches: (i) direct search and matching of MS/HRMS data in the MS/HRMS
Table 1 Stachybotrys species and strains used in the study for metabolite profiling and comparison

\begin{tabular}{lllll}
\hline Genus & Species & IBT no. & Chemotype & Origin \\
\hline Stachybotrys & chartarum & 7709 & $\begin{array}{c}\text { Macrocyclic trichothecene } \\
\text { producer (S) } \\
\text { Macrocyclic trichothecene } \\
\text { producer (S) }\end{array}$ & Building material, DK \\
Stachybotrys & chartarum & 9631 & Atranone producer (A) & DKA \\
Stachybotrys & chartarum & 7617 & Atranone producer (A) & Gypsum, DK \\
$\begin{array}{l}\text { Stachybotrys } \\
\text { Stachybotrys }\end{array}$ & $\begin{array}{l}\text { chartarum } \\
\text { chlorohalonata }\end{array}$ & 4466 & Atranone producer (A) & USA \\
Stachybotrys & chlorohalonata & 40295 & Atranone producer (A) & USA \\
\hline
\end{tabular}

IBT culture collection, author's address 
library and (ii) aggressive dereplication of the full HRMS data where searching was performed using lists of possible known compounds that have been described in the literature but not available as standards. The MS/HRMS library containing $\sim 1500$ compounds [36] included spectra from 25 reference standards originating from Stachybotrys, a further five tentatively identified Stachybotrys compounds and finally one tentatively identified in this study (stachybotrydial). A 300compound version of this library containing all Stachybotrys compounds is available at our WWW site [40].

Aggressive dereplication of the full HRMS data was created by extracting the Antibase2012 database for all compounds having Stachybotrys spp. as a source as well as recent references ( $\sim 140$ compounds) [10, 12, 22-24]. Adducts and common fragments included in this search function were $[\mathrm{M}+\mathrm{H}]^{+}$, $[\mathrm{M}+\mathrm{Na}]^{+},\left[\mathrm{M}+\mathrm{H}-\mathrm{H}_{2} \mathrm{O}\right]^{+}$and $\left[\mathrm{M}+\mathrm{NH}_{4}\right]^{+}$. All analysed ions were treated as being single charged; the area cut-off was set to 10,000 , and the mass spectrum was recorded below $10 \%$ of the peak maximum in order to avoid overloading the detector [36].

For fast screening of a larger number of samples, MassHunter Quantitative Analysis for QTOF (version B.06.00) was used [41]. The method for screening included all Stachybotrys compounds with known retention time, selecting the most abundant ion.

\section{Transferring the QTOF method to QqQ}

Analysis of selected Stachybotrys metabolites was performed on the Agilent Infinity 1290 UHPLC system coupled to an Agilent 6490 Triple Quadrupole (QqQ) mass spectrometer. Chromatographic separation was performed on an Agilent Poroshell 120 Phenyl-Hexyl column $(2.1 \times 100 \mathrm{~mm}, 2.7 \mu \mathrm{m})$ held at a temperature of $40{ }^{\circ} \mathrm{C}$ and at a flow of $0.4 \mathrm{~mL} / \mathrm{min}$. Both eluents contained $20 \mathrm{mM}$ formic acid in water (eluent $\mathrm{A}$ ) and in acetonitrile/2-propanol $(80 / 20, v / v)$ (eluent B). Additionally, $5 \mathrm{mM}$ ammonium formate was added to eluent A. The total analysis time was $10 \mathrm{~min}$, graduating from 20 to $80 \% \mathrm{~B}$ within $7 \mathrm{~min}$, increasing to $100 \% \mathrm{~B}$ in $0.1 \mathrm{~min}$, held for $1.1 \mathrm{~min}$, returning to $20 \% \mathrm{~B}$ in $0.1 \mathrm{~min}$ and maintaining until the end of the run. To avoid carry-over, the autosampler was operated in flow-through-needle mode and further coupled to an Agilent Flex cube which was used to perform series of flushing switching between main pass and bypass prior and after the injection at the flow of $4 \mathrm{~mL} / \mathrm{min}$. Precursor, product ion, fragmentor voltage and collision energy selection were based on the existing data previously obtained on the QTOF and entered in the MassHunter Personal Compound Database and Library (PCDL) Manager. This library contained information on fragmentation using 10, 20 and $40 \mathrm{eV}$ for each compound. After the initial selection of the precursor and product ion for each transition, the values for collision energies were further optimized around the selected value in order to achieve maximum sensitivity.
The fragmentor voltage was set to $380 \mathrm{~V}$ for all compounds/transitions. MassHunter Data Acquisition software version B06.01 was used to control the instrument. All analyses were performed only in $\mathrm{ESI}^{+}$mode using multiple reaction monitoring (MRM) acquisition. For each compound, two or three mass transitions were monitored with the dwell time set to 50 and cycle time of $1605 \mathrm{~ms}$. General source settings were gas temperature of $180^{\circ} \mathrm{C}$, gas flow of $12 \mathrm{~L} / \mathrm{min}$, sheath gas temperature of $350^{\circ} \mathrm{C}$, sheath gas flow of $12 \mathrm{~L} / \mathrm{min}$, nebulizer 20 psi $(137.9 \mathrm{kPa})$, capillary voltage of $3500 \mathrm{~V}$ and nozzle voltage of $0 \mathrm{~V}$ in positive mode.

The screening method for metabolites produced by indoor species other than Stachybotrys spp. was based on the work of Varga et al. [42]. Two mass transitions per compound, for nine compounds in total, were adopted and further adjusted for our instrument (Electronic Supplementary Material (ESM) Table S1).

\section{QqQ method validation}

For evaluation of method performance, external calibration of four quantitatively available standards (satratoxin $\mathrm{H}$, roridin E, stachybotrylactam and trichodermin) was performed as described in "Chemicals and standards". Linear non-weighed (satratoxin $\mathrm{H}$, roridin $\mathrm{E}$, stachybotrylactam) or $1 / x$ weighed (trichodermin) calibration curves were calculated by plotting the peak area of the analyte signal against the analyte concentration using Agilent MassHunter Quantitative Analysis for QqQ (version B.06.00). Limits of detection (LODs) and lower limits of quantification (LLOQs) were calculated at the lowest concentration levels of liquid standards as concentrations corresponding to a signal-to-noise ratio $(S / N)$ of 3/1 for LOD and 10/1 for LLOQ. $S / N$ was calculated using the "Calculate $\mathrm{S} / \mathrm{N}$ ratio" function in MassHunter Qualitative Analysis. The matrix effect was evaluated for dust samples, by extracting clean, unused Kimwipes ${ }^{\circledR}$ Lite wipes as described in "Extraction of pure cultures, biomass and dust". Blank extracts (solvents only) and wipes were spiked with a mixture of four standards in triplicates on five (satratoxin $\mathrm{H}$, roridin $\mathrm{E}$ ) or six (stachybo trylactam, trichodermin) concentration levels and analysed together with the blank extract as a control. This approach enabled direct determination of the matrix effect as the result of signal enhancement/suppression (SSE), by calculating slope ratios of the linear calibration functions $\operatorname{SSE}(\%)=[$ slope matrix - matched standards $/$ slope $\left._{\text {liquid standards }}\right] \times 100$.

\section{Results and discussion}

\section{Chemical analysis by UHPLC-QTOF}

Chemical analysis of extracts of fungal biomass (ESM, Table S2) collected from the kindergarten's gypsum 

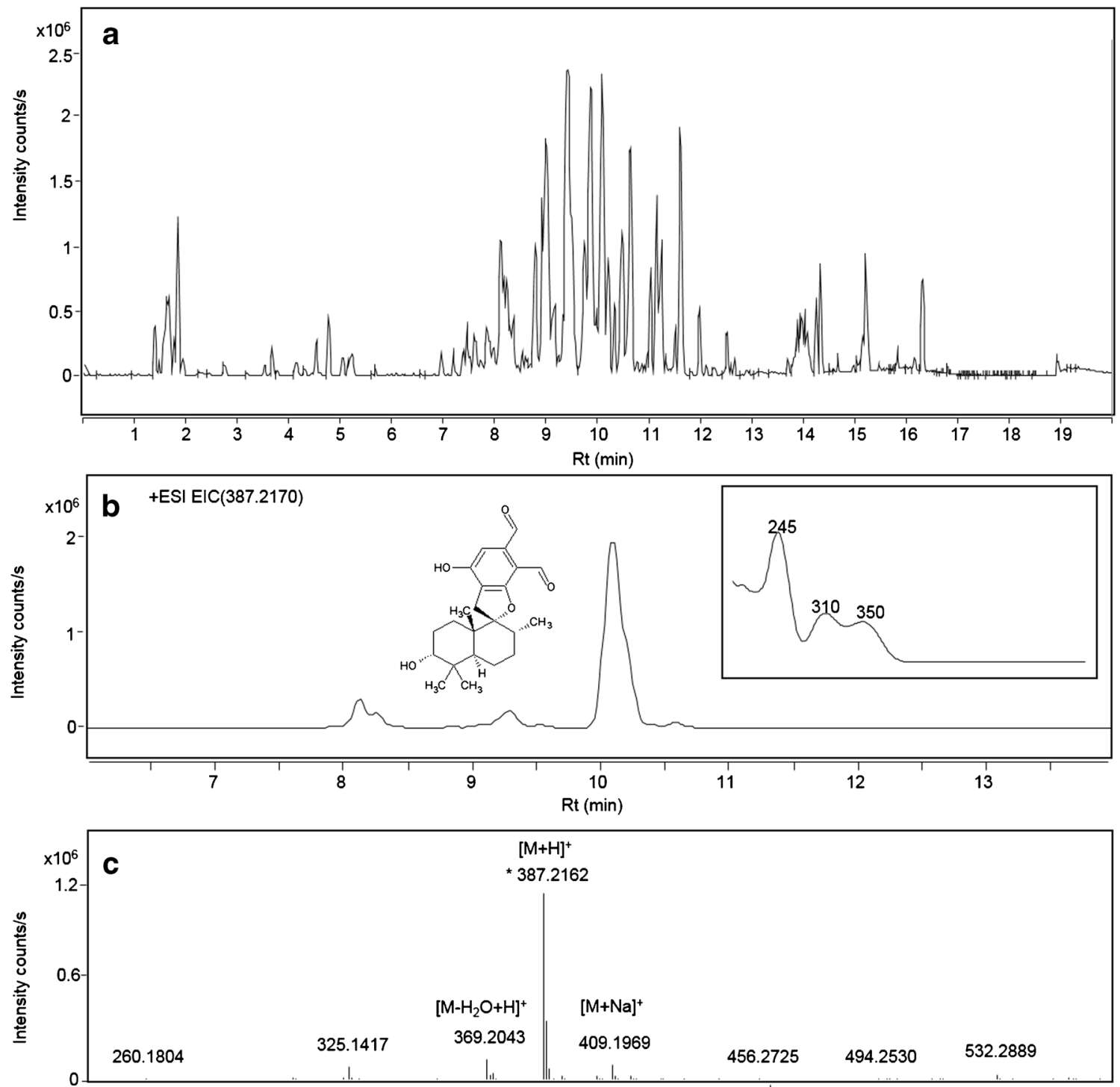

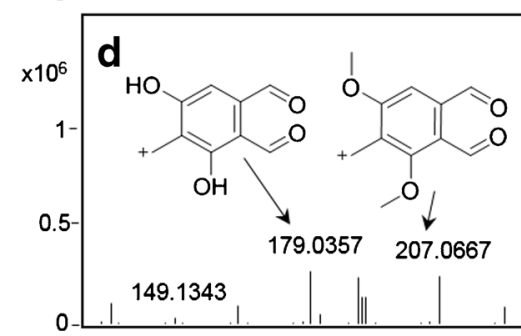

Fig. 2 Example of dereplication of stachybotrydial from data obtained on the QTOF: a base peak chromatogram (BPC) of $S$. chartarum pure culture (IBT 9631 on MEA) extract, b extracted ion chromatogram (EIC)

wallboard showed the presence of both atranones and macrocyclic trichothecenes. Further comparison of these extracts to metabolite profiles from Stachybotrys pure agar cultures confirmed the presence of both chemotypes. Whilst these results clearly suggested S. chartarum chemotype $\mathrm{S}$ as the macrocyclic trichothecene-producing contaminant, identification of the atranone-producing contaminant was more difficult, as chemical analysis could for the $\mathrm{m} / \mathrm{z}$ of 387.2166 with UV spectrum characteristic of stachybotrydial [31], c full scan spectrum, d MS/HRMS of stachybotrydial at $20 \mathrm{eV}$

not distinguish between $S$. chartarum chemotype A and $S$. chlorohalonata. Species-level identification of the atranone-producing contaminant would require isolation of the strain and classical morphological methods [9, 18], which were not performed in this study due to the time limitations.

Analysis of the gypsum wallboard extracts primarily revealed the presence of spirocyclic drimanes in all the analysed 
samples. Stachybotrydial and its reduced analogue Mer-NF5003-B (Fig. 1) were predominant in all analysed extracts. An example of stachybotrydial dereplication is presented in Fig. 2. The observed accurate mass $(\mathrm{m} / \mathrm{z} 387.2170,1 \mathrm{ppm}$ deviation) of the peak eluting at $10.1 \mathrm{~min}$ perfectly corresponded to the theoretical $\mathrm{m} / \mathrm{z}$ for the stachybotrydial molecular ion (387.2166), whose identity was further confirmed with the known UV spectrum for this compound [31, 42]. Furthermore, a full scan spectrum showed the presence of both the sodium adduct $[\mathrm{M}+\mathrm{Na}]^{+}$and dehydrated fragment $\left[\mathrm{M}-\mathrm{H}_{2} \mathrm{O}+\mathrm{H}\right]^{+}$, which validated the identification of the molecular ion. Finally, the presence of all the proposed fragments (ESM, Fig S1) in the MS/HRMS $20 \mathrm{eV}$ spectrum served as a final confirmation of stachybotrydial's identity. After confirming its identity, stachybotrydial was added to the library.

Two peaks with $m / z$ of 386.2326 (4 ppm deviation) and a retention time (RT) difference of $0.7 \mathrm{~min}$ were identified as stachybotrylactam in all extracts. No difference in fragmentation pattern at 10 and $20 \mathrm{eV}$ was found between the two compounds. Comparison with the stachybotrylactam standard identified the peak eluting at $8.35 \mathrm{~min}$ as the known compound, whilst a later-eluting compound will henceforth be referred to as the stachybotrylactam isomer. Library matching also identified a peak eluting at $8.1 \mathrm{~min}(\mathrm{~m} / \mathrm{z} 405.227,2.2 \mathrm{ppm}$ deviation) as Mer-NF-5003-B, as well as a peak eluting at
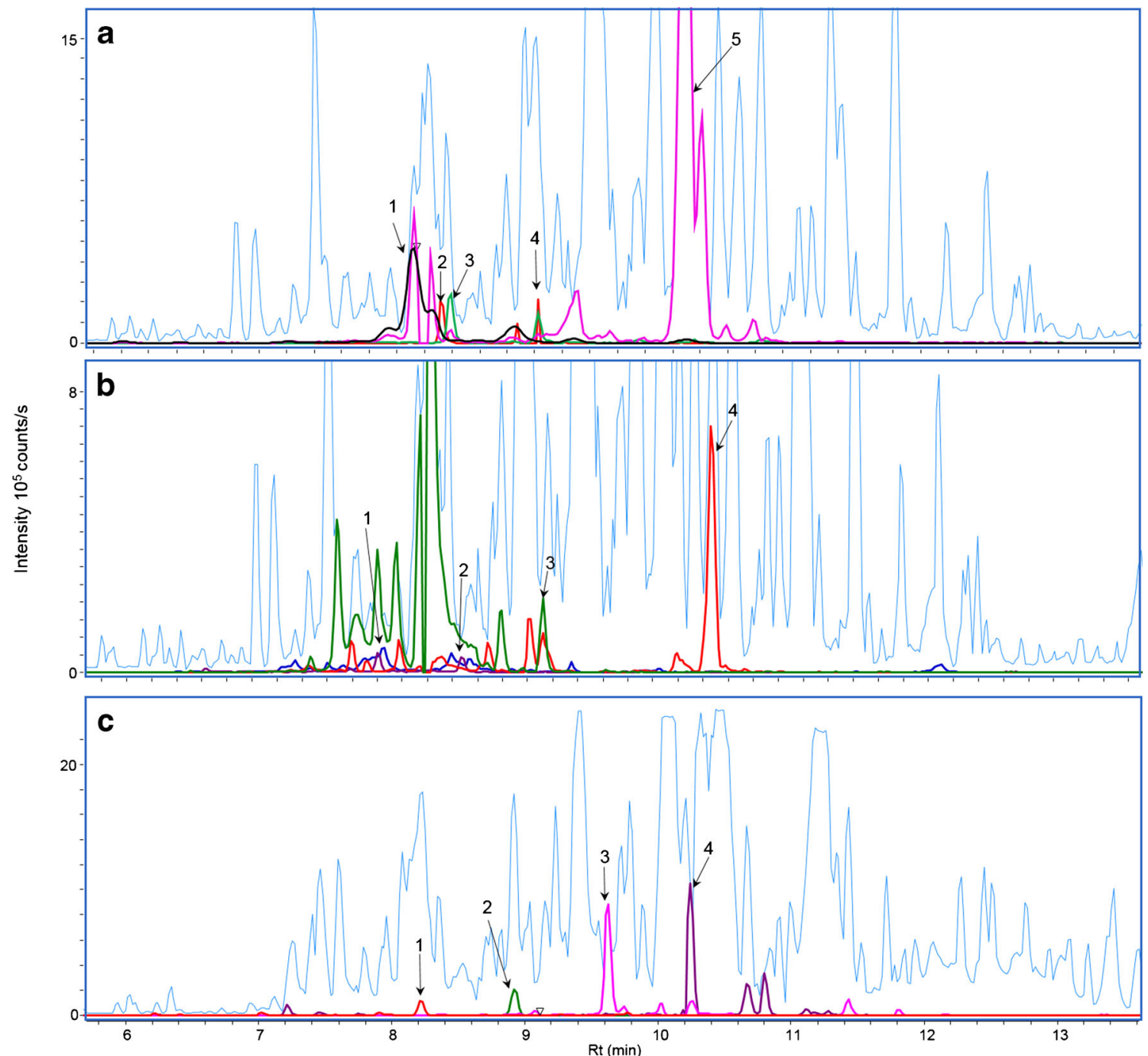

Fig. 3 Base peak chromatograms (BPC) of wall scraping (combination of wall swabs 3 and 4) from a contaminated wallboard analysed on QTOF merged with combined extracted ion chromatograms for a spirocyclic drimanes: $1 \mathrm{~m} / \mathrm{z} 405.227\left([\mathrm{M}+\mathrm{H}]^{+}\right.$, Mer-NF-5003-B) [43], $2 \mathrm{~m} / \mathrm{z}$ $430.2588\left([\mathrm{M}+\mathrm{H}]^{+}\right.$, stachybotryamide) $[44], 3 \mathrm{~m} / \mathrm{z} 386.2326\left([\mathrm{M}+\mathrm{H}]^{+}\right.$, stachybotrylactam) [42], $4 \mathrm{~m} / \mathrm{z} 386.2326\left([\mathrm{M}+\mathrm{H}]^{+}\right.$, stachybotrylactam isomer) and $\mathbf{5} \mathrm{m} / \mathrm{z} 387.2166\left([\mathrm{M}+\mathrm{H}]^{+}\right.$, stachybotrydial) [42]; b macrocyclic trichothecenes: $1 \mathrm{~m} / \mathrm{z}$ 546. $2695\left(\left[\mathrm{M}+\mathrm{NH}_{4}\right]^{+}\right.$, satratoxin $\left.\mathrm{H}\right)$, $2 \mathrm{~m} / \mathrm{z} 562.2645\left(\left[\mathrm{M}+\mathrm{NH}_{4}\right]^{+}\right.$satratoxin $\left.\mathrm{G}\right), 3 \mathrm{~m} / \mathrm{z} 548.2855\left(\left[\mathrm{M}+\mathrm{NH}_{4}\right]^{+}\right.$ roridin $\mathrm{L} 2)$ and $4 \mathrm{~m} / \mathrm{z} 532.2904\left(\left[\mathrm{M}+\mathrm{NH}_{4}\right]^{+}\right.$roridin $\left.\mathrm{E}\right)$; and $\mathbf{c}$ diterpenoids and their precursors: $1 \mathrm{~m} / \mathrm{z} 319.227\left([\mathrm{M}+\mathrm{H}]^{+}, 3,4\right.$-epoxy-6hydroxydolabella-7E,12-dien-14-one), $2 \mathrm{~m} / \mathrm{z} 303.2319$ ([M+H] $]^{+}, 6-$ hydroxydolabella-3E,7E,12-trien-14-one), $3 \mathrm{~m} / \mathrm{z} 417.227$ ([M+H $]^{+}$, atranone A) and $4 \mathrm{~m} / \mathrm{z} 447.238\left([\mathrm{M}+\mathrm{H}]^{+}\right.$, atranone B) 
$8.3 \mathrm{~min}(\mathrm{~m} / \mathrm{z} 430.2588,1.6 \mathrm{ppm})$ as stachybotryamide (Fig. 3). Both matches were further confirmed using UV spectra from authentic standards (ESM, Fig S2).

In four out of seven wall scrapings (ESM, Table S2) from the water-damaged bathroom, aggressive derepli cation suggested the presence of atranone $\mathrm{A}$ and $\mathrm{B}$, as well as $\left(1 S^{*}, 6 S^{*}, 11 S^{*}\right)$-6-hydroxydolabella-3E,7E,12trien-14-one (Fig. 3), which was further confirmed by authentic standards and UV/vis data [14] (ESM, Fig. S3). Furthermore, analysis of wallboard scrapings from the kindergarten not only showed the presence of spirocyclic drimanes and atranones but also yielded a positive hit for satratoxin $\mathrm{H}$, showing intense $\left[\mathrm{M}+\mathrm{NH}_{4}\right]^{+}$and $[\mathrm{M}+\mathrm{Na}]^{+}$as well as the MS/HRMS spectrum perfectly matching an authentic standard. Searching MS/HRMS data for $m / z 231.1300 \pm 0.0100$, a characteristic fragment of trichothecenes [14], confirmed not only the presence of satratoxin $\mathrm{H}$ but also the presence of other trichothecenes (roridin L2, satratoxin G, roridin E, Fig S4, ESM), which were also verified by comparison to authentic standards. The compound with an RT of 10.45 min possessed the characteristic fragment with $\mathrm{m} / \mathrm{z} 231.1384$ and also showed the presence of $\mathrm{m} / \mathrm{z} 361.2003$ characteristic of roridin E [14], as well as the precursor ion of $\mathrm{m} / \mathrm{z}$ 532.2904 (1.3 ppm deviation) matching $\left[\mathrm{M}+\mathrm{NH}_{4}\right]^{+}$for roridin E. In this sample, the intensities of the trichothecenes with respect to the intensities of the spirocyclic drimanes were significantly (three- to fivefold) lower, increasing the risk of overlooking these compounds in the presence of drimanes. The problem of detection of atranones, dolabellanes and macrocyclic trichothecenes in the presence of spirocyclic drimanes is well known [12, 31]. Jarvis et al. [12] developed a normal phase SPE method (PEI silica) for fractionation of the $S$. chartarum extracts in spirocyclic drimanes and macrocyclic trichothecenes or atranones. Due to the possible loss of the target analyte and time associated with fractionation, we decided to attempt to increase the sensitivity of our method for the low-abundant compounds by transferring the method to the more sensitive and selective UHPLC-QqQ.

\section{UHPLC-QTOF to UHPLC-QqQ method transfer}

In contrast to the UHPLC-QTOF method, the UHPLC-QqQ gradient was started at $20 \%$ organic phase (eluent B) instead of $10 \%$, a shorter column was used and there was a steeper increase to $100 \%$ acetonitrile/2-propanol (eluent B) to elute compounds faster and as sharper peaks. In order to provide sufficient precursor ion intensity and suppress the formation of $[\mathrm{M}+\mathrm{Na}]^{+}$(which gave poor daughter ion yields) for macrocyclic trichothecenes and promote $\left[\mathrm{M}+\mathrm{NH}_{4}\right]^{+}$formation, ammonium formate was added to eluent A. For each macrocyclic trichothecene, both mass transition with molecular ion $[\mathrm{M}+\mathrm{H}]^{+}$and ammonium adduct $\left[\mathrm{M}+\mathrm{NH}_{4}\right]^{+}$as precursor ion were used, thus ensuring reliable identification (Table 2).
Table 2 List of Stachybotrys metabolites included in QqQ method with optimized ESI-MS/ MS

\begin{tabular}{|c|c|c|c|c|}
\hline Metabolite & Rt (min) & $\begin{array}{l}m / z \\
\text { precursor } \\
\text { ion }\end{array}$ & Ion species & $\begin{array}{l}m / z \text { product ion } \\
(\text { collision energy }(\mathrm{V}))\end{array}$ \\
\hline Satratoxin $\mathrm{H}^{\mathrm{a}}$ & 3.60 & $\begin{array}{l}546 \\
\mathbf{5 2 9}\end{array}$ & $\begin{array}{l}{\left[\mathrm{M}+\mathrm{NH}_{4}\right]^{+}} \\
{[\mathrm{M}+\mathrm{H}]^{+}}\end{array}$ & $\begin{array}{l}249(15) / 231(15) \\
\mathbf{2 3 1 ( 1 5 )}\end{array}$ \\
\hline Satratoxin $\mathrm{G}^{\mathrm{a}}$ & 3.50 & $\begin{array}{l}562 \\
545\end{array}$ & $\begin{array}{l}{\left[\mathrm{M}+\mathrm{NH}_{4}\right.} \\
{[\mathrm{M}+\mathrm{H}]^{+}}\end{array}$ & $\begin{array}{l}249(15) / 231(15) \\
\mathbf{2 3 1 ( 1 5 )}\end{array}$ \\
\hline Roridin $\mathrm{L}^{\mathrm{a}}$ & 4.20 & $\begin{array}{l}548 \\
\mathbf{5 3 1}\end{array}$ & $\begin{array}{l}{\left[\mathrm{M}+\mathrm{NH}_{4}\right]^{+}} \\
{[\mathrm{M}+\mathrm{H}]^{+}}\end{array}$ & $\begin{array}{l}249(15) / 231(15) \\
\mathbf{2 3 1 ( 1 5 )}\end{array}$ \\
\hline Roridin $\mathrm{E}^{\mathrm{a}}$ & 5.10 & $\begin{array}{l}\mathbf{5 3 2} \\
515\end{array}$ & $\begin{array}{l}{\left[\mathrm{M}+\mathrm{NH}_{4}\right]^{+}} \\
{[\mathrm{M}+\mathrm{H}]^{+}}\end{array}$ & 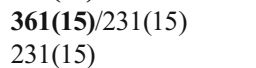 \\
\hline Atranone $\mathrm{A}^{\mathrm{b}}$ & 4.60 & 417 & {$[\mathrm{M}+\mathrm{H}]^{+}$} & $381(10) / 357(15)$ \\
\hline Atranone $\mathrm{B}^{\mathrm{b}}$ & 5.40 & 447 & {$[\mathrm{M}+\mathrm{H}]^{+}$} & $387(10) / 369(10)$ \\
\hline Dolabellane $^{\mathrm{a}, \mathrm{b}, \mathrm{c}}$ & 3.90 & 303 & {$[\mathrm{M}+\mathrm{H}]^{+}$} & $219(15)$ \\
\hline Stachybotrylactam $^{\mathrm{a}}$ & 4.10 & 386 & {$[\mathrm{M}+\mathrm{H}]^{+}$} & $\begin{array}{l}256(20) / \mathbf{1 7 8}(\mathbf{3 5}) / \\
150(40)\end{array}$ \\
\hline $\begin{array}{l}\text { Stachybotrylactam } \\
\text { isomer }\end{array}$ & 3.70 & 386 & {$[\mathrm{M}+\mathrm{H}]^{+}$} & $256(20) / 178(35)$ \\
\hline Stachybotryamide & 4.10 & 430 & {$[\mathrm{M}+\mathrm{H}]^{+}$} & $\mathbf{2 6 0 ( 3 0 ) / 2 2 2 ( 2 0 )}$ \\
\hline Stachybotrydial & 4.90 & 387 & {$[\mathrm{M}+\mathrm{H}]^{+}$} & $207(20) / 179(20)$ \\
\hline Mer-NF-5003-B & 3.65 & 405 & {$[\mathrm{M}+\mathrm{H}]^{+}$} & $387(15) / 369(20)$ \\
\hline Trichodermin $^{\mathrm{a}}$ & 3.85 & 293 & {$[\mathrm{M}+\mathrm{H}]^{+}$} & $143(10) / \mathbf{1 0 9}(15)$ \\
\hline
\end{tabular}

Precursor ion-to-quantifier ion mass transition together with collision energy used is presented in bold

${ }^{\text {a }}$ Reference standard available

${ }^{\mathrm{b}}$ Not quantified due to the unavailability of the quantitative standard with the same/similar structure

${ }^{\mathrm{c}}\left(1 S^{*}, 3 R^{*}, 4 R^{*}, 6 S^{*}, 11 S^{*}\right)-3,4-E$ poxy-6-hydroxydolabella-7E,12-dien-14-one 
Mass transition giving the most abundant signal was chosen as the quantifier ion, regardless of whether the chosen precursor was the proton adduct $[\mathrm{M}+\mathrm{H}]^{+}$or ammonium adduct $[\mathrm{M}+$ $\left.\mathrm{NH}_{4}\right]^{+}$. Since all the compounds included in the method eluted between 3 and $5.5 \mathrm{~min}$, the retention time window for all transitions was set to $4 \mathrm{~min}$ (from 2 to $6 \mathrm{~min}$ ). With the dwell time for each transition of $50 \mathrm{~ms}$, the duty cycle of $1605 \mathrm{~ms}$ and the typical peak width of $20 \mathrm{~s}$, we obtained a minimum of 12 data points across peak, which was enough to ensure reliable peak integration [45].

Collision energies, chosen through comparison with the QTOF MS/HRMS spectra obtained at 10, 20 and $40 \mathrm{eV}$, only required minimal optimization (changes of less than $5 \mathrm{eV}$ ) in most cases. This demonstrates the value of the information that can be obtained from full scan QTOF experiments, as well as that acceptable QqQ methods can be obtained by direct library-to-QqQ transfer.

Transitions and starting collision energies for chosen metabolites included in the method detecting secondary metabolites produced by indoor fungi other than Stachybotrys were adopted from Varga et al. [42]. Further optimization of this method only included collision energy adjustment within a few volts (ESM, Table S1). The fragmentor voltage $(380 \mathrm{~V})$ cannot be altered between compounds as it is fixed by the ionfunnel instrument, but far less important than on a cone-based instrument.

\section{UHPLC-QqQ method validation}

The matrix effect was calculated as the slope ratio of the linear functions for matrix-matched standards and liquid standards and is presented in Table 3. The matrix effect was evaluated only for one matrix, clean Kimwipes ${ }^{\circledR}$ Lite wipes used to collect dust. This matrix was chosen due to the fact that dust needed to be extracted together with the wipes. Among evaluated metabolites, both satratoxin $\mathrm{H}$ and roridin $\mathrm{E}$ showed enhancement of the signal, which was minor for roridin E (4\%) and significantly higher for satratoxin $\mathrm{H}(34 \%)$. In contrast, signals for stachy botrylactam and trichodermin were suppressed by 11 and $21 \%$ respectively. Evaluation of blank matrix extract showed no signal for any of the tested analytes. Clearly, it can be expected that dust itself will further suppress/ enhance the signals obtained; however, one should note that the dust samples taken were very low and taken from places cleaned several times per week, and not behind furniture or other places rarely cleaned. Vishwanth et al. [26] reported a severe matrix effect in dust for the majority of the target analytes; however, the reported enhancement for stachybotrylactam by $6 \%$ was negligible, whilst the matrix effect for roridin $E$, satratoxin $H$ and trichodermin was not reported. Undoubtedly, the observed enhancement and suppression in our study showed that
Table 3 Matrix effect, signal suppression/enhancement (SSE), standard error of regression line (Sy), average observed accuracy (Acc) and maximum observed accuracy $\left(\mathrm{Acc}_{\max }\right)$, coefficients of variation $(\mathrm{CV})$, limit of detection (LOD) and lower limit of quantification (LLOQ) values for Stachybotrys metabolites

\begin{tabular}{|c|c|c|c|c|c|c|}
\hline Metabolite & $\operatorname{SSE}^{\mathrm{d}}(\%)$ & $\mathrm{Sy}^{\mathrm{e}}$ & $\begin{array}{l}\operatorname{Acc}\left(\operatorname{Acc}_{\max }\right)^{\mathrm{f}} \\
(\%)\end{array}$ & $\mathrm{CV}^{\mathrm{g}}(\%)$ & $\begin{array}{l}\mathrm{LOD}^{\mathrm{h}} \\
\left(\mathrm{ng} / \mathrm{cm}^{2}\right)\end{array}$ & $\begin{array}{l}\operatorname{LLOQ}^{\mathrm{i}} \\
\left(\mathrm{ng} / \mathrm{cm}^{2}\right)\end{array}$ \\
\hline Satratoxin $\mathrm{H}^{\mathrm{a}}$ & 134 & 1.8 & $28(79)$ & 2.2 & 15 & 50 \\
\hline Satratoxin $\mathrm{G}^{\mathrm{a}}$ & 134 & 1.8 & $28(79)$ & 2.2 & 15 & 50 \\
\hline Roridin $E^{\mathrm{b}}$ & 104 & 4.9 & 29 (114) & 2.2 & 0.1 & 0.2 \\
\hline Roridin $\mathrm{L} 2^{\mathrm{b}}$ & 104 & 4.9 & 29 (114) & 2.2 & 0.1 & 0.2 \\
\hline Stachybotrylactam $^{\mathrm{c}}$ & 89 & 36 & $21(77)$ & 2.5 & 2 & 6 \\
\hline Stachybotrylactam isomer ${ }^{\mathrm{c}}$ & 89 & 36 & $21(77)$ & 2.5 & 2 & 6 \\
\hline Stachybotryamide $^{\mathrm{c}}$ & 89 & 36 & $21(77)$ & 2.5 & 2 & 6 \\
\hline Stachybotrydial $^{\mathrm{c}}$ & 89 & 36 & $21(77)$ & 2.5 & 2 & 6 \\
\hline Mer-NF-5003-B ${ }^{c}$ & 89 & 36 & $21(77)$ & 2.5 & 2 & 6 \\
\hline Trichodermin & 79 & 40 & $13.4(28)$ & 4.4 & 5 & 17 \\
\hline
\end{tabular}

${ }^{\text {a }}$ Compounds calibrated against satratoxin $\mathrm{H}$ standard

${ }^{\mathrm{b}}$ Compounds calibrated against roridin E standard

${ }^{\mathrm{c}}$ Compounds calibrated against stachybotrylactam standard

${ }^{\mathrm{d}} \mathrm{SSE} \%$ - signal suppression/enhancement calculated as the slope ratio of the linear functions for matrix-matched standards and liquid standards multiplied by 100

${ }^{\mathrm{e}}$ Standard error of the regression line

${ }^{\mathrm{f}}$ Average accuracy on all levels - accuracy on each level was calculated as the ratio of the calculated concentration and the expected concentration multiplied by 100 . Acc $c_{\max }$ represents the maximum observed value regardless of the level including the values outside allowed limits $(20 \%)$

${ }^{\mathrm{g}} \mathrm{CV} \%$ - calculated as the average of the ratios of the standard deviation and average concentration multiplied by 100 for each level

${ }^{\mathrm{h}}$ LOD - the limit of detection calculated at the lowest concentration levels as concentrations corresponding to a signal-to-noise ratio $(S / N)$ 3/1

${ }^{\mathrm{i}} \mathrm{LLOQ}$ - lower limit of quantification calculated at the lowest concentration levels as concentrations corresponding to a signal-to-noise ratio $(S / N) 10 / 1$ 


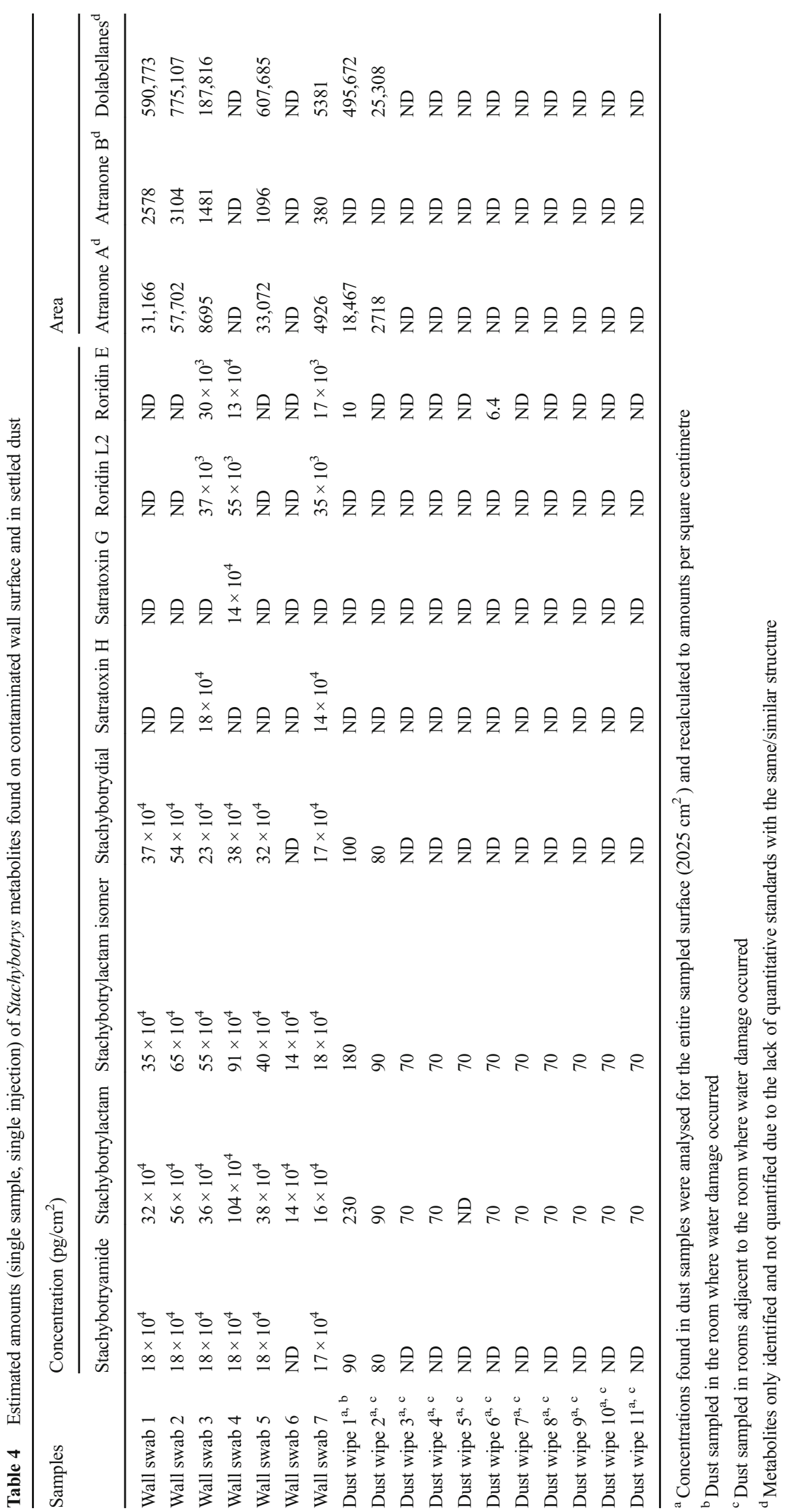


matrix-matched calibration is not adequate to account for the matrix effect and that the use of isotopic-labelled internal standards would be preferable [26, 42].

The linear range covered three orders of magnitude for stachybotrylactam and trichodermin, but only two orders of magnitude for satratoxin $\mathrm{H}$ and roridin $\mathrm{E}$, due to the limited quantities of these two standards. All constructed calibration curves showed good linearity across the entire range, and given the expected amounts in dust, this is considered sufficient. Calculated coefficients of variation (CV \%) for both liquid and matrix-matched standards were generally low, with the highest for trichodermin ( $8 \%$ for liquid standards and $4 \%$ for matrix-matched standards). Non-weighed calibration curves were chosen for stachybotrylactam, roridin $\mathrm{E}$ and satratoxin $\mathrm{H}$, due to the higher resulting linearity and the fact that the concentrations in analysed samples tended to shift towards the middle and higher ranges of the constructed curves. Use of non-weighed calibration curves resulted in accuracy outside of the allowed limits (20\%) on the lowest level; however, the observed accuracy on all other levels was within limits (Table 3). It should be noted that dust was sampled and analysed on a much larger surface $(45 \times 45 \mathrm{~cm})$ and later recalculated to the amount per square centimetre; therefore, the obtained concentrations in all samples are comparable. In the absence of quantitative standards for all mycotoxins included in the method, stachybotryamide, stachybotrydial and Mer-NF-5003-B were quantified based on the calibration curve for stachybotrylactam. Similarly, roridin L2 was quantified based on the calibration curve constructed for roridin E, whilst satratoxin $G$ was quantified based on the calibration curve for satratoxin $\mathrm{H}$. This approach was obviously based on similarity in structures, although we are well aware that similar structures often show differences in fragmentation patterns and intensities of created fragments. Although possessing different elemental compositions and thereby different precursor ions, all macrocyclic trichothecenes produce same fragments, namely $\mathrm{m} / \mathrm{z} 231$ and 249. Only roridin E exhibits specific mass transition $(\mathrm{m} / \mathrm{z} 532$ to 361), which was chosen for quantification.

The situation was more complicated in the case of the spirocyclic drimanes, where the structural differences between compounds were greater, most notably in the lack of 2-pyrrolidone moiety in stachybotrydial and Mer-NF5003-B in comparison to stachybotrylactam and stachy botryamide (Fig. 1). Clearly, calculated concentrations, especially in cases where structural differences between metabolites were more prominent, are likely to be more inaccurate; however, since reference standards for all drimanes were not commercially available, we found that it was a better option to calibrate against stachy botrylactam which is commercially available.

\section{Wall swabs and dust wipe analyses by QqQ}

The UHPLC-QqQ method was used to analyse dust wipes and wall swabs collected in the water-damaged kindergarten. The method was able to detect Stachybotrys compounds in settled dust, both in the infected bathroom and in the adjacent rooms, and further differentiate two co-existing chemotypes. The results showing all detected metabolites are presented in Table 4.

The results confirmed the presence of both macrocyclic trichothecene- (chemotype $\mathrm{S}$ ) and atranone-producing contaminants (chemotype A) in both wall and dust samples. In two out of seven wall swab samples, both trichothecene mycotoxins and atranones/dolabellanes were detected in the same sample (wallboard swabs 3 and 7). In other cases, there was either trichothecenes or atranones/dolabellanes present in the wall swabs, depending on the sampling site on the wallboard. This shows the coexistence of two chemotypes on the same material, with no clear borderline between areas of their contamination. Among trichothecenes, the highest amounts $\left(180 \mathrm{ng} / \mathrm{cm}^{2}\right)$ were estimated for satratoxin $\mathrm{H}$, whilst atranones/dolabellanes could not be quantified due to the insufficient amounts of the standard. No trichodermin was found either in dust wipe or wall swab samples. Spirocyclic drimanes represented by stachybotrylactam, stachybotrydial and stachybotryamide were dominant in all samples as expected, based on results obtained on the QTOF. The exception was Mer-NF-5003-B, whose production on the materials was reduced compared to pure cultures. Estimated concentrations for drimanes in samples collected with Q-tips were within one order of magnitude (135-1042 ng/ $/ \mathrm{cm}^{2}$ ) with the total amount for all drimanes collected with the Q-tip estimated to be $2.5 \mu \mathrm{g} / \mathrm{cm}^{2}$. Screening for metabolites produced by species other than Stachybotrys revealed the presence of roquefortine $\mathrm{C}$ and meleagrin. This suggests the presence of Penicillium chrysogenum on sampled material, which is among species most commonly isolated from indoor environment [46].

For the dust wipe samples, all groups of metabolites (trichothecenes, atranones/dolabellanes, drimanes) were detected in settled dust collected in the bathroom where water damage occurred (dust 1, Fig. 4). Settled dust collected in adjacent rooms contained, in most cases, stachybotrylactam and stachybotrylactam isomer. Roridin $\mathrm{E}$ was found only in one sample in the concentration of $6.4 \mathrm{pg} / \mathrm{cm}^{2}$, whilst dolabellanes were found in two samples; however, the peaks were of very low intensity. Neither meleagrin nor roquefortine $\mathrm{C}$ was found in the dust wipe samples. The amounts of mycotoxins in dust wipes in comparison to the amounts found in the wall swabs were three to four orders of magnitude lower, both in the cases of drimanes and trichothecenes $\left(600 \mathrm{pg} / \mathrm{cm}^{2}\right.$ of total drimanes in the bathroom with the water damage and highest estimated total amount of drimanes of $340 \mathrm{pg} / \mathrm{cm}^{2}$ in the adjacent room). Although stachybotrylactam has been 
Fig. $4 \mathrm{MS} / \mathrm{MS}$ transitions for all mycotoxins identified in dust sample collected in the waterdamaged room
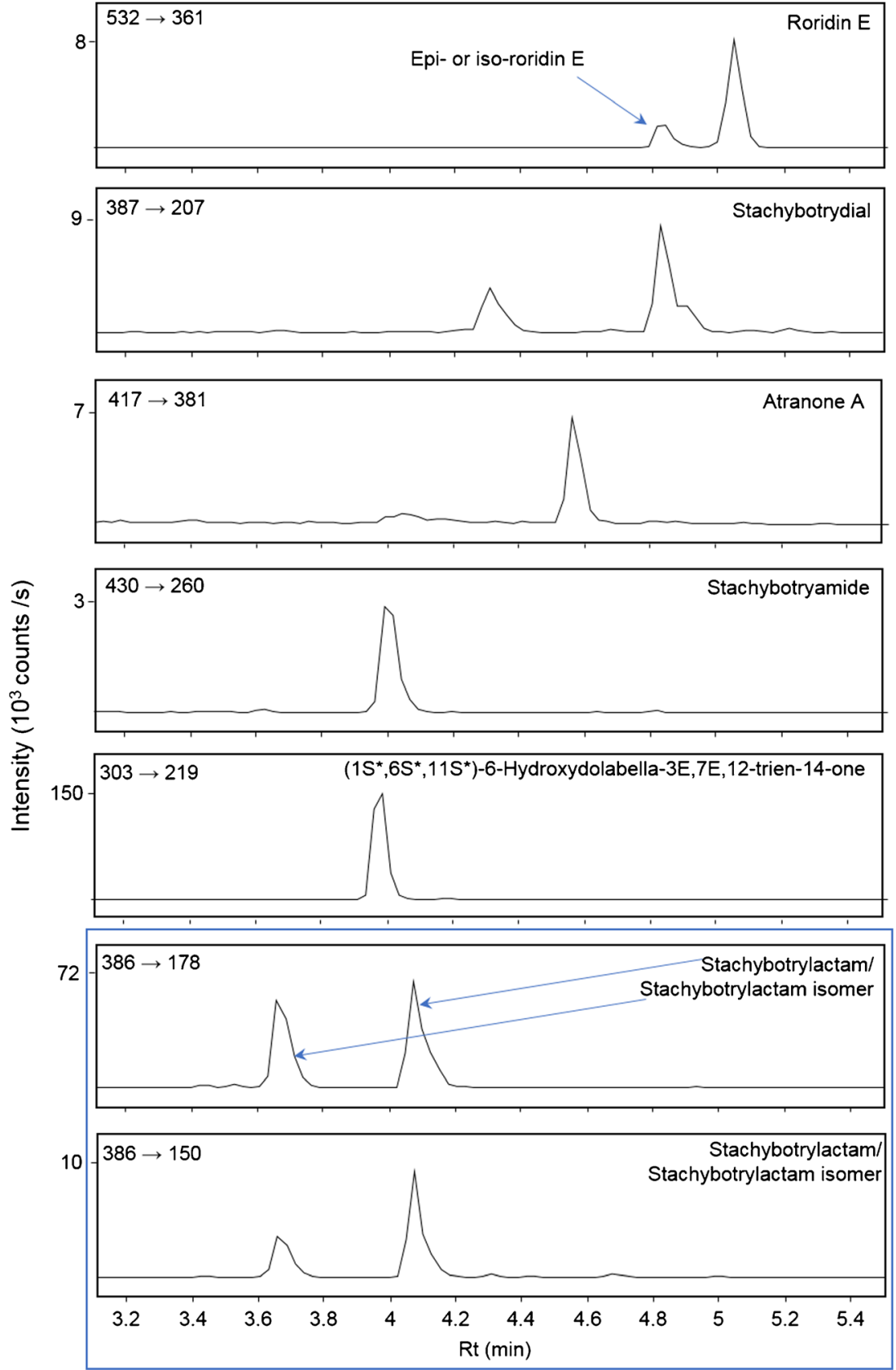

reported in dust samples previously, its presence has only been found in samples collected from waste management facilities [47] and in settled dust collected in Dutch schools (in only $1 \%$ of the collected samples, [48]). In addition to this, attempts have been made to quantify macrocyclic trichothecenes in dust; however, only the simple trichothecenes verrucarol and trichodermol (hydrolysis products of the satratoxins) analysed by GC-MS have been reported [30, 48, 49]. Furthermore, Polizzi et al. [27] reported the analysis of roridin E on contaminated material but not in dust. To the best of our knowledge, this is the first study where macrocyclic trichothecenes have been found directly in settled dust collected from a water-damaged building. It is also the first time that a comparison between the amounts of mycotoxins produced on contaminated wall surfaces and the amounts of mycotoxins that actually become airborne has been made. Moreover, the presence of drimanes in dust samples collected in rooms adjacent to the room with water damage clearly demonstrates a wide distribution of mycotoxins in detectable amounts and this is promising for their use as biomarkers.

The mycotoxin content of settled dust is important for overall exposure assessment. The presented method appeared to be a useful tool for identifying and estimating the amounts of indoor contaminants in dust. This indicates that the future focus could primarily be analysis of dust samples. Given that dust is the primary route of exposure to these mycotoxins, but 
the detection here has previously proven difficult, this method may provide an effective means of gathering considerable chemical data and improve our understanding of mycotoxin toxicity in contaminated buildings.

\section{Conclusion}

This case study presents a semi-quantitative UHPLC-QqQ method with several new exposure biomarkers, which was developed based on UHPLC-qQTOF screening of culture extracts. The method was able to identify 12 Stachybotrys metabolites of which four could be quantified based on authentic standards and an additional six estimated based on similar compounds. The method was applied to samples collected in a water-damaged building contaminated by $S$. chartarum chemotype $\mathrm{S}$, as well as with atranone-producing contaminant (S. chartarum chemotype A or S. chlorohalonata). The obtained results represent a step forward in solving the problems of exposure to mycotoxins in damp indoor environments, detecting for the first time the presence of the same mycotoxins on contaminated gypsum wallboard surfaces and in settled dust. Furthermore, the method enables fast estimation of the mycotoxin's amounts in analysed samples. Demonstrating that method transfer from UHPLC-qQTOF to UHPLC-QqQ instruments is facile means that this method can be supplemented with additional biomarkers in subsequent exposure studies. This methodology represents a significant advance in the detection of mycotoxins in dust samples, which is the main route of exposure leading to toxicity and illness. As such, this study could have an impact on our understanding of the relationship between mould exposure and sickness. Further work includes expanding this methodology to include mycotoxins and biomarkers produced by other species and genera of indoor fungi and further case studies to demonstrate the reliability and applicability of this method.

Acknowledgments The authors would like to thank the VILLUM Foundation for financial support and Agilent Technologies for the Thought Leader Donation of the UHPLC-qTOF and UHPLC-QqQ systems.

\section{Compliance with ethical standards}

Conflict of interest The authors declare that they have no conflict of interest.

Open Access This article is distributed under the terms of the Creative Commons Attribution 4.0 International License (http:// creativecommons.org/licenses/by/4.0/), which permits unrestricted use, distribution, and reproduction in any medium, provided you give appropriate credit to the original author(s) and the source, provide a link to the Creative Commons license, and indicate if changes were made.

\section{References}

1. Johanning E, Biagini RE, Hull D, Morey PR, Jarvis BB, Landsbergis P. Health and immunology study following exposure to toxigenic fungi (Stachybotrys chartarum) in a water damaged office environment. Int Arch Occup Environ Health. 1996;68:207-18.

2. Täubel M, Sulyok M, Vishwanath V, Bloom E, Turunen M, Järvi K, et al. Co-occurence of toxic bacterial and fungal secondary metabolites in moisture-damaged indoor environments. Indoor Air. 2011;21:368-75.

3. Miller JD, McMullin DR. Fungal secondary metabolites as harmful indoor air contaminants: 10 years on. Appl Microbiol Biotechnol. 2014;98:9953-66.

4. Croft WA, Jarvis BB, Yatawara CS. Airborne outbreak of trichothecene mycotoxicosis. Atmos Environ. 1986;20:549-52.

5. Jarvis BB, Sorenson WG, Hintikka E-L, Nikulin M, Zhou Y, Jiang $\mathrm{J}$, et al. Study of toxin production by isolates of Stachybotrys chartarum and Memnoniella echinata isolated during a study of pulmonary hemosiderosis in infants. Appl Environ Microbiol. 1998;64:3620-5.

6. Etzel RA. Indoor and outdoor air pollution: tobacco smoke, moulds and diseases in infants and children. Int J Hyg Environ Health. 2007;210:611-6.

7. Jarvis BB, Lee Y-W, Comezoglu SN, Yatawara CS. Trichothecenes produced by Stachybotrys atra from Eastern Europe. Appl Environ Microbiol. 1986;51:915-8.

8. Forgacs J, Carll WT. Mycotoxicosis. Adv Vet Sci. 1962;7:273-93.

9. Andersen B, Nielsen KF, Thrane U, Szaro T, Taylor JW, Jarvis BB. Molecular and phenotypic descriptions of Stachybotrys chlorohalonata sp nov and two chemotypes of Stachybotrys chartarum found in water-damaged buildings. Mycologia. 2003;95:1227-38.

10. Nielsen KF. Mould growth on building materials: secondary metabolites, mycotoxins and biomarkers. 2002; PhD Thesis, Technical University of Denmark.

11. Nielsen KF, Huttunen K, Hyvärinen A, Andersen B, Jarvis BB, Hirvonen MR. Metabolite profiles of Stachybotrys isolates from water-damaged buildings and their induction of inflammatory mediators and cytotoxicity in macrophages. Mycopathologia. 2001;154:201-5.

12. Hinkley SF, Jarvis BB. Chromatographic method for Stachybotrys toxins. In: Pohland A, Trucksess MW, editors. Methods molecular biology, Mycotoxin protocols, vol. 157. Totowa: Humana Press Inc; 2000. p. 173-94.

13. Andersen B, Frisvad JC, Søndergaard I, Rasmussen IS, Larsen LS. Associations between fungal species and water damaged building materials. Appl Environ Microbiol. 2011;77:4180-8.

14. Nielsen KF. Mycotoxin production by indoor molds. Fungal Genet Biol. 2003;39:103-17.

15. Nikulin M, Reijula K, Jarvis BB, Hintikka EL. Experimental lung mycotoxicosis induced by Stachybotrys atra. Int J Exp Pathol. 1997;77:213-8.

16. Nielsen KF, Holm G, Uttrup LP, Nielsen PA. Mould growth on building materials under different water activities. Influence of humidity and temperature on fungal growth and secondary metabolism. Int Biodeterior Biodegrad. 2004;54:325-36.

17. Flannigan B, Miller JD. Microbial growth in indoor environments. In: Flannigan B, Samson RA, Miller JD, editors. Microorganisms in home and indoor work environments. Diversity, health impacts, investigation and control. 2nd ed. Boca Raton: CRC Press; 2011. p. 57-107.

18. Samson RA, Houbraken J, Thrane U, Frisvad JC, Andersen B. Food and indoor fungi. Utrecht: CBS-KNAW-Fungal Biodiversity Centre; 2010. p. 1-398. 
19. Brasel TL, Douglas DR, Wilson SC, Straus DC. Detection of airborne Stachybotrys chartarum macrocyclic trichothecene mycotoxins on particulates smaller than conidia. Appl Environ Microbiol. 2005;71:114-22.

20. Gorny RL, Reponen T, Willeke K, Schmechel D, Robine E, Boissier M, et al. Fungal fragments as indoor air biocontaminants. Appl Environ Microbiol. 2002;68:3522-31.

21. Kildesø J, Wurtz H, Nielsen KF, Kruse P, Wilkins K, Thrane U, et al. Determination of fungal spore release from wet building material. Indoor Air. 2003;13:148-55.

22. Li Y, Wu C, Liu D, Proksch P, Guo P, Lin W, et al. Phenylspirodrimanes from the sponge-associated fungus Stachybotrys chartarum with antihyperlipidemic activities. J Nat Prod. 2013;77:138-47.

23. Ma X, Li L, Zhu T, Ba M, Li G, Gu Q, et al. Phenylspirodrimanes with anti-HIV activity from the sponge-derived Stachybotrys chartarum MXH-X73. J Nat Prod. 2013;76:2298-306.

24. Wu B, Oesker V, Wiese J, Malien S, Schmaljohann R, Imhoff JF. Spirocyclic drimanes from the marine fungus Stachybotrys sp strain MF347. Mar Drugs. 2014;12:1924-38.

25. Gottschalk C, Bauer J, Meyer K. Detection of satratoxin $\mathrm{G}$ and $\mathrm{H}$ in indoor air from a water-damaged building. Mycopathologia. 2008; 166:103-7

26. Vishwanath V, Sulyok M, Labuda R, Bicker W, Krska R. Simultaneous determination of 186 fungal and bacterial metabolites in indoor matrices by liquid chromatography/tandem mass spectrometry. Anal Bioanal Chem. 2009;395:1355-72.

27. Polizzi V, Delmulle B, Adams A, Picco AM, Rosseel Y, Kindt R, et al. JEM spotlite: fungi, mycotoxins and microbial volatile organic compounds in moldy interiors from water damaged buildings. J Environ Monit. 2009;11:1849-58.

28. Brasel TL, Martin JM, Carriker CG, Wilson SC, Straus DC. Detection of airborne Stachybotrys chartarum macrocyclic trichothecene mycotoxins in the indoor environment. Appl Environ Microbiol. 2005;71:7376-88.

29. Dietrich R, Johanning E, Gareis M, Schneider E, Usleber E, Märtlbauer E. Immunochemical detection of mycotoxins associated with stachybotrytoxicosis. In: Johanning E, editor. Bioaerosols, fungi and mycotoxins: health effects, assessment, prevention and control. Anonymous. New York: Eastern New York Occupational \& Environmental Health Center; 1999. p. 482-91.

30. Bloom E, Bal K, Nyman E, Must A, Larsson L. Mass spectrometrybased strategy for direct detection and quantification of some mycotoxin produced by Stachybotrys and Aspergillus spp. in indoor environments. Appl Environ Microbiol. 2007;73:4211-7.

31. Andersen B, Nielsen KF, Jarvis BB. Characterization of Stachybotrys from water-damaged buildings based on morphology, growth and metabolite production. Mycologia. 2002;94:392-403.

32. Nielsen KF, Smedsgaard J. Fungal metabolite screening: database of 474 mycotoxins and fungal metabolites for dereplication by standardized liquid chromatography-UV-mass spectrometry methodology. J Chromatogr A. 2003;1002:111-36.

33. Herebian D, Zuhlke S, Lamshoft M, Spiteller M. Multi-mycotoxin analysis in complex biological matrices using LC-ESI/MS: experimental study using triple stage quadrupole and LTQ-Orbitrap. J Sep Sci. 2009;32:939-48.

34. Klitgaard A, Iversen A, Andersen MR, Larsen TO, Frisvad JC, Nielsen KF. Aggressive dereplication using UHPLC-DAD-
QTOF: screening extracts for up to 3000 fungal secondary metabolites. Anal Bioanal Chem. 2013;406:1933-43.

35. Broecker S, Herre S, Wust B, Zweigenbaum J, Pragst F. Development and practical application of a library of CID accurate mass spectra of more than 2500 toxic compounds for systematic toxicological analysis by LC-QTOF-MS with data-dependent acquisition. Anal Bioanal Chem. 2011;400:101-17.

36. Kildgaard S, Månsson M, Došen I, Klitgaard A, Frisvad JC, Larsen $\mathrm{TO}$, et al. Accurate dereplication of bioactive secondary metabolites from marine-derived fungi by UHPLC-DAD-QTOFMS and a MS/ HRMS library. Mar Drugs. 2014;12:3681-705.

37. Renaud JB, Kelman MJ, Qi TYF, Seifert KA, Sumarah MW Product ion filtering with rapid polarity switching for the detection of all fumonisins and AAL-toxins. Rapid Commun Mass Spectrom. 2015;29:2131-9.

38. Smedsgaard J. Micro-scale extraction procedure for standardized screening of fungal metabolite production in cultures. J Chromatogr A. 1997;760:264-70.

39. Došen I, Nielsen KF, Clausen G, Andersen B. Potentially harmful secondary metabolites produced by indoor Chaetomium species on artificially and naturally contaminated building materials. Indoor Air. 2016. doi:10.1111/ina.12290.

40. DTU Stachybotrys mycotoxin-fungal secondary metabolite MS/ HRMS library. Available online: http://www.bio.dtu.dk/english/ Research/Platforms/Metabolom/MSMSLib. Accessed on 07 Dec 2015.

41. Nielsen KF, Larsen TO. The importance of mass spectrometric dereplication in fungal secondary metabolite analysis. Front Microbiol. 2015;6:1-15.

42. Varga E, Glauner T, Berthiller F, Krska R, Schmacher R, Suluyok M. Development and validation of a (semi-)quantitative UHPLCMS/MS method for the determination of 191 mycotoxins and other fungal metabolites in almonds, hazelnuts, peanuts and pistachios. Anal Bioanal Chem. 2013;405:5087-104.

43. Jarvis BB. Stachybotrys chartarum: a fungus for our time. Phytochemistry. 2003;64:53-60.

44. Ayer WA, Miao S. Secondary metabolites of the aspen fungus Stachybotrys cylindrospora. Can J Chem. 1993;71:487-93.

45. Commission Decision No. 2002/657/EC of 12 August 2002 implementing Council Directive 96/23/EC concerning the performance of analytical methods and the interpretation of results - consolidated version 10 Jan 2004. http://eur-lex.europa.eu/legalcontent/EN/TXT/PDF/?uri=CELEX:32002D0657\&from=EN Accessed 07 Dec 2015

46. Nielsen KF, Gravesen S, Nielsen PA, Andersen B, Thrane U, Frisvad JC. Production of mycotoxins on artificially and naturally infested building materials. Mycopathologia. 1999;145:43-56.

47. Vishwanath V, Sulyok M, Weingart G, Kluger B, Täubel M, Mayer $\mathrm{S}$, et al. Evaluation of settled floor dust for the presence of microbial metabolites and volatile anthropogenic chemicals in indoor environments by LC-MS/MS and GC-MS methods. Talanta. 2011;85: 2027-38.

48. Peitzsch M, Sulyok M, Täubel M, Vishwanath V, Krop E, BorrasSantos A, et al. Microbial secondary metabolites in school buildings inspected for moisture damage in Finland, the Netherlands and Spain. J Environ Monit. 2012;14:2044-53.

49. Bloom E, Grimsley LF, Pehrson C, Lewis J, Larsson L. Molds and mycotoxins in dust from water-damaged homes in New Orleans after hurricane Katrina. Indoor Air. 2009;19:153-8. 